\title{
The Effect of Heel Height of Shoe on Ankle Muscle Activation Pattern in Women with Functional Ankle Instability during Stair Descending
}

\author{
F. Ghaderi ${ }^{1}$, E. Shahmoradi ${ }^{1}$, M. Moghadam Salimi ${ }^{1,2}$, M. Asghari Jafarabadi ${ }^{3,4}$, \\ S. Goljarian ${ }^{1}$ \\ 1 Department of Physical Therapy, Faculty of Rehabilitation, Tabriz University of Medical Sciences, Tabriz, Iran \\ 2 Department of Neuroscience and Cognition, Faculty of Advanced Sciences, Tabriz University of Medical \\ Sciences, Tabriz, Iran \\ 3 Road Traffic Injury Research Center, Tabriz University of Medical Sciences, Tabriz, Iran \\ ${ }^{4}$ Department of Statistics and Epidemiology, Faculty of Health, Tabriz University of Medical Sciences, Tabriz, Iran
}

\section{CORRESPONDING AUTHOR:}

Maryam Moghadam Salimi

Department of Physical therapy

Faculty of Rehabilitation

Tabriz University of Medical Sciences

29 Bahman Bulvar

Tabriz, Iran

E-mail: moghadamm@tbzmed.ac.ir

DOI:

10.32098/mltj.04.2021.08

LEVEL OF EVIDENCE: 3B

\begin{abstract}
SUMMARY
Background. Wearing high-heeled shoes (HHS) increases the risk of musculoskeletal disorders, especially in unstable situations. This study was conducted to evaluate the electromyographic (EMG) activity of the ankle muscles during stair descent with HHS in women with and without functional ankle instability (FAI).

Methods. In this experimental study, Thirty-six subjects were surveyed in 4 groups: The group 1: Ten non- habitual HHS wearer with FAI, group 2: Eight habitual HHS wearer with FAI, group 3: Ten non-habitual HHS wearer without FAI, and group 4: Eight habitual HHS wearer without FAI. The EMG activity was obtained from the tibialis anterior (TA), peroneus longus (PL), and gastrocnemius medialis (GM) muscles during stair descent with HHS and barefoot.

Results. In non-habitual and habitual FAI group, onset latency of the TA and PL muscles was longer in the HH compared to barefoot. However, in healthy groups, onset latency of the selected muscles was longer only in the non-habitual healthy subjects. In the non-habitual FAI group there was a significant increase in TA and PL muscles onset latency compared to that in the non-habitual healthy group. EMG activity of the TA, PL and GM muscles were significantly greater in the HHS group compared to the barefoot in FAI and healthy groups during stair descending.

Conclusions. The findings of this study suggest that wearing HHS may put people with FAI and without the experience of wearing HHS at greater risk for developing ankle sprains.
\end{abstract}

\section{KEY WORDS}

Functional ankle instability; surface electromyography; high-heeled shoes; stair walking.

\section{INTRODUCTION}

Wearing high-heeled shoes (HHS) makes walking unstable $(1,2)$ and may place individuals at greater risk for imbalance and falling $(3,4)$. Excessive ankle plantar flexion due to wearing HHS, changes in kinematics, and kinetics of walking $(3,5,6)$. Long-term use of HHS limits ankle range of motion (7) and changes the ankle muscle activation pattern (8). It also could increase the muscular activation and load- ing, which in turn can accelerate muscle fatigue and joint instability $(1,2,5-7)$. Wearing HHS could be more likely harmful during less stable situations such as walking on uneven surfaces or stair ascending- descending.

Stair walking is a common activity of daily life that demands greater muscle activation of the lower extremities compared with level walking $(9,10)$. During Stair ascending-descending, single-leg support time is longer (11), and it needs more muscle control and activation. So, fatigue and discoordina- 
tion of the muscles may subsequently happen. This condition could raise the risk of imbalance and fall, particularly with HHS $(12,13)$. In fact, the excessive plantar flexion coupling with the foot inversion, especially in stair descent, is likely to increase the possibility of instability and lateral ankle sprain with HHS (14).

Most of the studies that investigated the stair walking activities with HHS, have been focused on healthy individuals. Though millions of women wear HHS only a small number of them get involved in ankle injuries or falling (1). Therefore, the purpose of this study was to determine the effects of wearing HHS on electromyography (EMG) activity of the ankle muscles during stair descent in women with and without FAI and with various experiences of wearing HHS. Two hypotheses were tested: 1) during stair descending with HHS, people with FAI would show different muscle activation pattern when compared with healthy subjects, 2) during stair descending with HHS, habitual HHS wearers would show different muscle activation pattern when compared with non-habitual HHS wearers.

\section{MATERIALS AND METHODS}

\section{Subjects}

\section{Study design}

Through a public announcement, 36 women (age range, 20-33 years) with recurrent chronic lateral ankle sprain, were recruited in this cross-sectional study. Volunteers were included if they had more than one ankle sprain in their dominant leg within the last year, and with the initial incidence more than one year before the study. Self-reported functional conditions determined and confirmed by foot and ankle ability measure (FAAM) questionnaire, and participants with the score of less than $85 \%$ were recruited (14). The sample size of the study was estimated utilizing primary information from a pilot study on the ankle sprain in two different heights of shoes (mean1 $=1.88, \mathrm{SD} 1=0.68$; mean2 $=0.81, \mathrm{SD} 2=0.51$ ). Taking into account $80 \%$ power, $95 \%$ confidence, a two-tailed test the sample size was calculated to be at least 7 subjects per group, by G-Power Software (15). Considering a dropout rate of about $20 \%$, the sample size increased to 9 participants per group.

All subjects completed a questionnaire consisting of their demographic information, medical history, and the time period they wore HHS in the past six months. Subjects were allocated to 4 groups based on the ankle health status (FAI or healthy) and the frequency of using HHS (non-habitual or habitual). The inclusion criteria for the FAI were a history of more than one ankle sprain in their dominant leg and the score of less than $85 \%$ in FAAM (14). Group one consisted of 10 subjects with FAI and experience of wearing HHS less than 10 times, each less than two hours, in the past 1 year (non-habitual FAI). Group 2 consisted of 8 subjects with FAI who habitually wore HHS at least 2 times a week, every 4 hours in the past 1 year (habitual FAI). Group 3 consisted of 10 subjects with healthy ankles and similar experience in using HHS as group one (non-habitual healthy). Group 4 consisted of 8 subjects with healthy ankles and similar experience in using HHS as group 2 (habitual healthy). The minimum heel height of the shoes the subjects used was 5 $\mathrm{cm}$. The healthy groups 3 and 4 were matched according to age, height, weight, body mass index (BMI), and dominant leg with the FAI groups 1 and 2, respectively. The exclusion criteria were pregnancy, any neurological or musculoskeletal symptoms in the three months prior to data collection and any dysfunction which may preclude them from safe ambulation in shoes with up to $8 \mathrm{~cm}$ high heel, obese subjects (BMI> 30), any history of surgery in the musculoskeletal structures of the lower extremity or back, a history of fracture in the lower extremity, circulatory disorder, and serious joint conditions such as rheumatoid arthritis and osteoarthritis.

This study was approved by the regional ethical committee of Tabriz University of Medical Sciences, Code number: IR.TBZMEED.REC.1399.489. All volunteers were explained the study procedure and signed a written informed consent if they were agreeing to participant. The study was conducted according to the international ethical standards as well as the ethical principles of the Muscle, Ligaments and Tendons Journal (16).

\section{Equipment}

Electromyography signals were recorded from the tibialis anterior (TA), peroneus longus (PL), and gastrocnemius medialis (GM) using surface EMG equipment (ME6000, Mega electronics Ltd, Finland). The EMG electrodes (Ag/ $\mathrm{AgCl}$ ) were placed over the muscle bellies and aligned with the longitudinal axis of the muscles with a center-to-center distance of $2 \mathrm{~cm}$. One reference electrode was placed over the lateral malleolus. The cables and interfaces were shielded to eliminate interference. Raw EMG signals were recorded at the sampling rate of $1,500 \mathrm{~Hz}$, amplified (differential amplifier, CMRR $=96 \mathrm{~dB}$, gain 1,000, noise $<1 \mathrm{uV}$ ), filtered (using Butter-worth filter, effctive band-width 1-500 Hz), converted with A/D board of 14 bit, stored in a PC computer, and analyzed by Megawin software.

\section{Data processing}

To determine the onset latency of each muscle, a threshold of two standard deviations from the mean value (mean 
+ 2 SD) observed at baseline was calculated using Megawin software. The onset points were controlled visually to confirm the validity of the calculated points. The relative differences in the onset times between each muscle and the first activated muscle (i.e., GM) were calculated. All EMG data were averaged over 3 repetitions of each test. Root mean square (RMS) of raw EMG signals calculated with $2 \mathrm{~ms}$ sliding window and EMG signal amplitude of each muscle was expressed as a percentage of the $3 \mathrm{~s}$ maximum RMS values obtained during a maximal voluntary isometric contraction (MVIC) test for $5 \mathrm{~s}$.

\section{Subject preparation and procedure}

Prior to data collection, the subjects performed several practice trials to get familiar with the task. Each subject was provided footwear in their respective shoe size and completed two practice trials for each $\mathrm{HH}$ to check for proper shoe fit and comfort. Subjects' skin was then prepared for electrode placement by shaving, cleaning with $70 \%$ alcohol, and slightly abrading the area with fine sandpaper. Electrode placement followed the guidelines as reported by Perotto et al. as follows (17):

- TA: the electrodes were placed four finger-breadths below the tibial tuberosity and one finger-breadth lateral to the tibial crest.

- PL: the electrodes were placed three finger-breadths below the fibular head directed toward the lateral aspect of the fibula.

- GM: the electrodes were placed as far as a palm below the popliteal fold on the medial side of the calf muscle. The electrode placement is presented in figure $\mathbf{1}$.

Raw EMG data were rectified, smoothed, and filtered prior to processing. After rectification, the EMG signal amplitude was normalized to the maximal activity level recorded during the trial. For normalization purposes, before the performance of the test, $5 \mathrm{~s}$ of the EMG data were recorded three times for each muscle, while the subjects performed MVIC. For the TA muscle, the subjects were in a supine position and performed maximum dorsiflexion with inversion, with the hallux in neutral position (not extended). The PL muscle was also assessed in supine position with the subjects performing eversion with plantar flexion. The GM muscle was assessed with the subjects in a prone position performing maximum plantar flexion.

EMG signals of the muscles were recorded at the dominant leg in healthy groups and at the injured dominant leg in FAI groups. The experimental staircase consisted of three steps (step height $18 \mathrm{~cm}$ and tread length $30 \mathrm{~cm}$ ). The subjects stood on the third step of the staircase and were instructed to descend

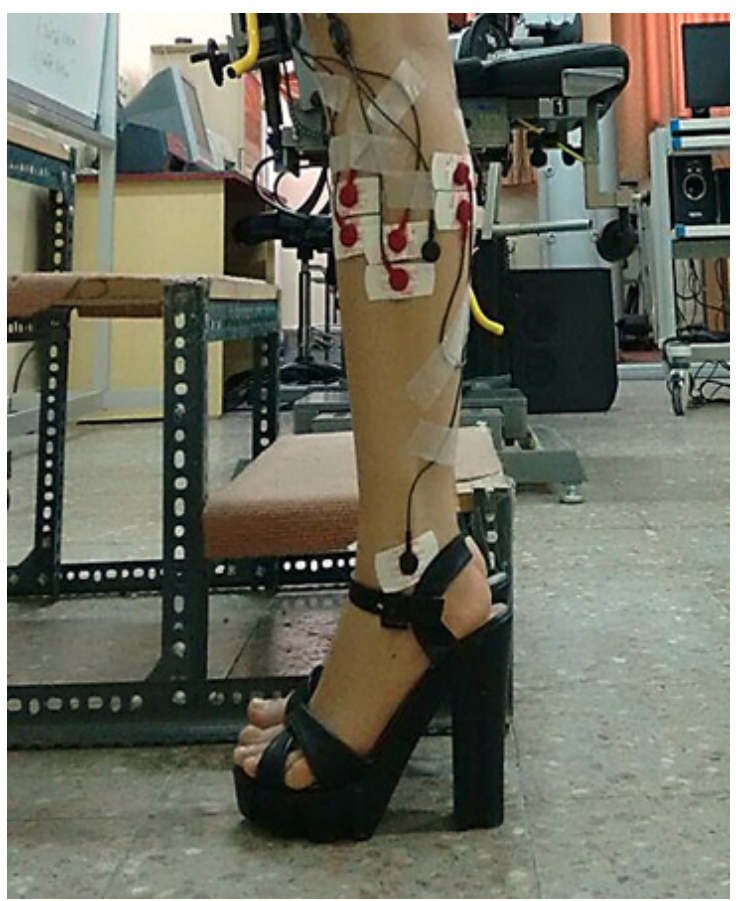

Figure 1. Electrode placement with high heeled shoe. Tibialis Anterior (TA): four finger-breadths below the tibial tuberosity and one finger-breadth lateral to the tibial crest, Peroneus longus (PL): three finger-breadths below the fibular head directed toward the lateral aspect of the fibula. Gastrocnemius medialis (GM): as far as a palm below the popliteal fold on the medial side of the calf muscle.

the stairs at a self-selected pace with barefoot and then with HHS $(8 \mathrm{~cm}$ heel height with $3.5 \mathrm{~cm}$ diameter of the heel for each shoe), placing only one foot on each step (step-over-step) with arms freely moving at their sides. During stair descent, the stride cycle was defined by the dominant foot beginning at foot contact on the third step and ending at subsequent contact of the same foot on the first step. Each trial was performed three times, with the maximum amplitude of trials being averaged. To avoid muscle fatigue in the lower limbs, a 2-minute rest period was given between each trial.

\section{Statistical analysis}

In this study, according to the correlation structure among measurements, a Mixed Model test and an estimation procedure of Restricted Max Likelihood (REML) was utilized with the help of a first order autoregressive covariance structure (AR (1)). The age, weight, height, BMI data were expressed as the mean $\pm \mathrm{SE}$, and analysis of variance (ANOVA) and chi-square tests for dominant leg were performed to identify statistically significant differences between groups. The 
Table I. Baseline demographic characteristics (mean \pm SE) of the groups.

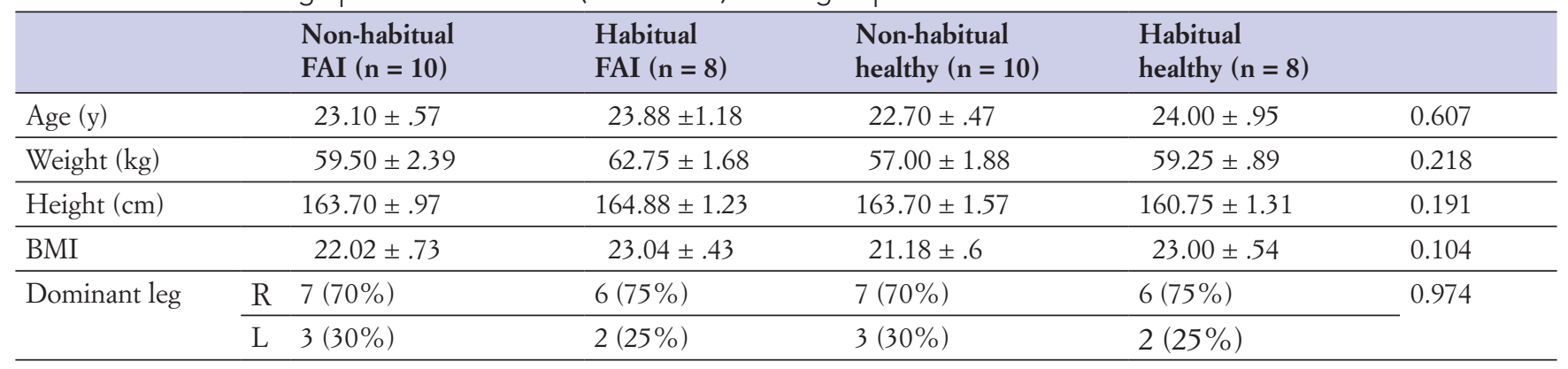

significant level of $\mathrm{P}=0.05$ and confidence interval of $95 \%$ were taken into account by applying SPSS software, version 16 to analyze the data.

\section{RESULTS}

Table I displays the baseline demographic characteristics of the subjects of the present study. No statistically significant differences were found among the groups regarding the demographics.

\section{EMG onset time}

The overall EMG onset latency during stair descent is illustrated in figure $\mathbf{2} \mathbf{a}, \mathbf{b}$. In FAI groups (non-habitual and habitual FAI) and healthy subjects (non-habitual and habitual healthy). In FAI group, the onset latency of the TA and PL muscles was longer in the HHS wearers compared to barefoot during stair descent $(\mathrm{p}<0.0001)$.

However, in healthy groups, onset latency of the TA and PL muscles was longer only in the non-habitual healthy subjects $(p=0.001)$ with no significant increase in onset latency in habitual healthy subjects $(p>0.05)$.

In the non-habitual FAI group, there was a statistically significant increase in TA $(p=0.002)$ and PL $(p=0.001)$ muscles onset latency compared to that in non-habitual healthy group. The non-habitual FAI group displayed a significant increase in muscle onset latency for TA $(p=0.002)$ and PL $(\mathrm{p}=0.022)$ muscles compared to the habitual FAI group.

\section{EMG amplitude}

Average RMS values of the TA, PL and GM muscles activity was found to be statistically greater in the HHS group compared to the barefoot in FAI and healthy groups when descending the stairs $(\mathrm{p}<0.0001)$.

No significant differences were noted between habitual and non-habitual groups (healthy and FAI) for average RMS values in selected muscles during stair descend ( $p>0.05)$.

A comparison of RMS of EMG signals among all groups illustrated in figures $3,4,5$.
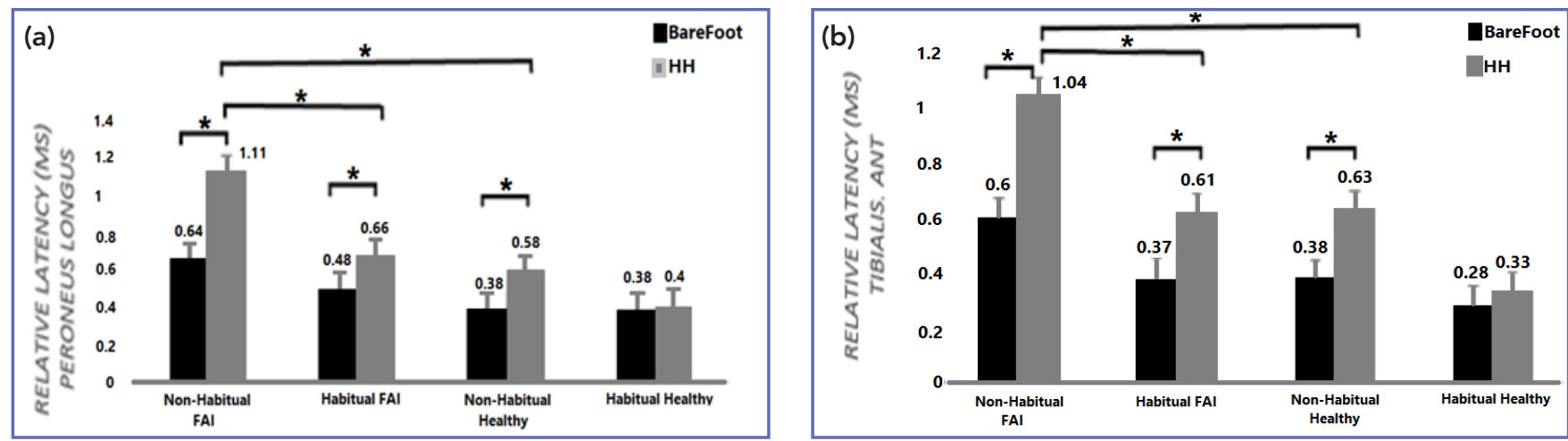

Figure 2. Between group changes (mean \pm SE) in relative latency of the TA and PL. (a) Between group changes (mean \pm SE) in relative latency of the TA muscle compared to GM muscle during stair descent. (b) Between group changes (mean \pm SE) in relative latency of the PL muscle compared to GM muscle during stair descent.

*Significant difference between two groups $(\mathrm{p} \leq 0.05)$ according to Mixed Model test. 


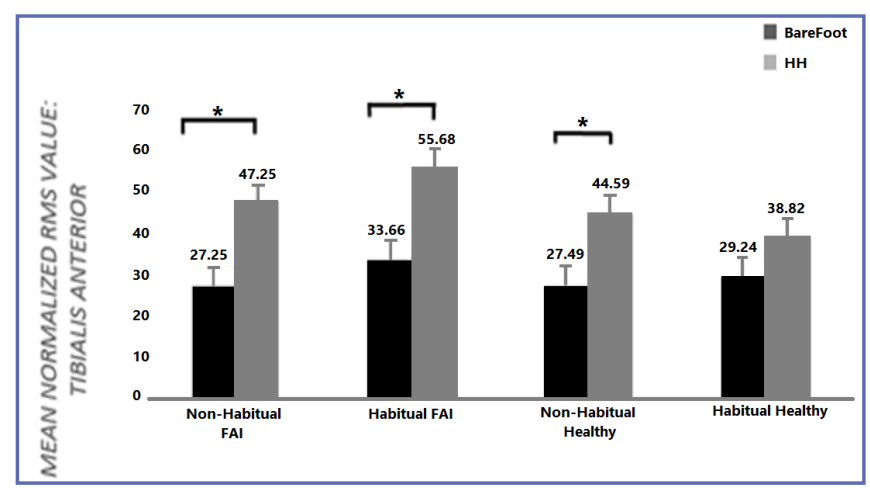

Figure 3. Between group changes (mean \pm SE) in normalized RMS values (mean \pm SE) of the TA muscle during stair descent.

*Significant difference between two groups $(\mathrm{p} \leq 0.05)$ according to Mixed Model test.

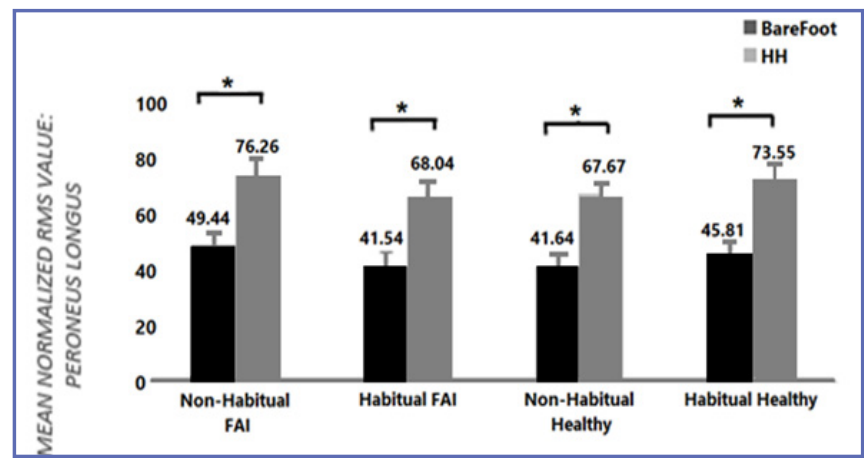

Figure 4. Between group changes (mean \pm SE) in normalized RMS values of the PL muscle during stair descent.

*Significant difference between two groups $(\mathrm{p} \leq 0.05)$ according to Mixed Model test.

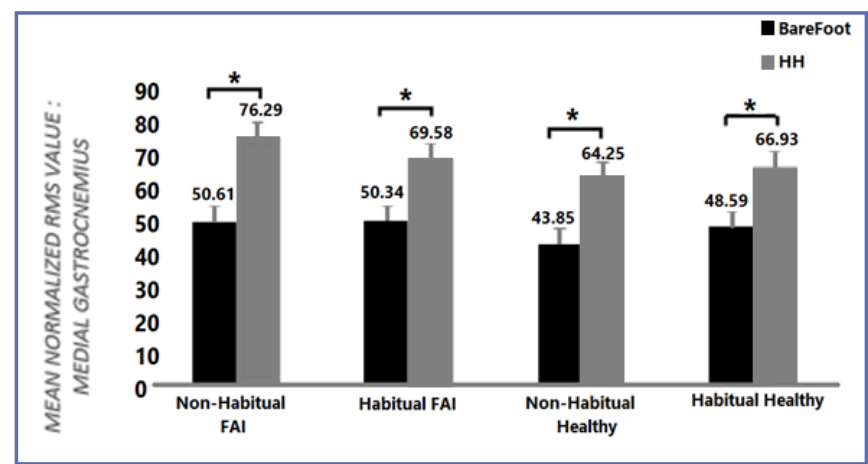

Figure 5. Between group changes (mean $\pm \mathrm{SE}$ ) in normalized RMS values of GM muscle during stair descent.

*Significant difference between two groups $(\mathrm{p} \leq 0.05)$ according to Mixed Model test.

\section{DISCUSSION}

Stair walking with HHS is an unstable situation which places the individual at greater danger of imbalance and falling (10). Risk of ankle inversion injuries (14), especially in subject with FAI could be increase during stair ascending-descending. The aim of the present study was to investigate the effects of the wearing HHS on EMG activity of the ankle muscles in the patient with FAI and during stair descending. We also examined the effect of the HHS wearing experience on muscle activity pattern. The muscle onset latency and electrical activity of the TA, PL, and GM, were measured in healthy subjects and patients with FAI. The result revealed that the muscle onset latency of the selected muscles, increased in non-habitual FAI, habitual FAI, and non-habitual healthy groups during stair descent with HHS compared to the barefoot or low-heeled shoes. This change may contribute to the lengthening of the tibiofemoral lever arm resulted from the lengthening of the lower limb and altered ankle posture $(11,20)$.

The result was also revealed that irrespective of the experience of waring HHS, during stair descending the onset latency of the muscles in FAI groups was prolonged than that in the healthy subjects. According to the previous studies, in healthy group excessive plantar flexion and decrease in the base of support due to wearing HHS increase the ankle eversion moment and changes the activity pattern of the muscles to stabilizing the joint and foot arc during different phases of the gate $(13,18)$. However, ankle sprain could cause damage to the joint structures and create some changes in muscle mechanoreceptors and $\gamma$ motor neurons. These changes may lead to a reduction of the muscle spindle sensitivity and augmentation of the muscle onset latency, which appears to be demonstrated by the present study $(19,20)$.

The study presented that muscle activation pattern in healthy, non-habitual HHS wearers was prolonged compared with that in habitual HHS wearers during stair descending. According to the Herzog et al. (2003), muscles are the primary contributors after alteration in lower limb biomechanics (21). Wearing HHS is associated with biomechanical changes in ankle structure, joint reaction force and center of pressure specially in stair descending (22). So, wearing HHS could alter the muscle activation pattern in HHS wearers (18). However, the habitual healthy group presented similar muscle activation pattern with HHS to the BF. It implies that long term use of $\mathrm{HH}$, likely contributes to the constant feed-forward postural response to the central nervous system (CNS) $(23,24)$ and subsequently could change the body postural reaction. It seems that following longer use of the HHS, the CNS may adapt to the change of the footwear and could adopt different movement strategy 
of the habitual HHS wearers $(1,25)$. In a study by Aljear et al. (2012), it was suggested that the controlled HHS walking in healthy habitual HHS wearers rely more on an increased movement variability, as a neural strategy which is adapted by the CNS to maintain balance (1).

In the present study, wearing HHS caused a significant increase of TA, PL, and GM muscle activation in both healthy subjects and the patients with FAI. In accordance with our findings, Kermani et al. (2018) and Arnadottir et al. (2011), showed similar results for the PL and GM muscle activation in healthy subjects $(26,27)$. Isokinetic measurement of the ankle muscle during wearing high heeled also presented strengthening of these muscles in HHS wearers compare to the barefoot (28). This increase in muscle activation is presumably related to the plantar flexion position of the ankle during activities with high heel. In fact, the ankle plantar flexion is mechanically an unstable situation, so the increased activation might overcome the instability while wearing HHS. Thus, increased muscle activity is expected partly due to less stability at the talocrural joint and the smaller body base of support while walking on high heel (26). Su et al. (2012), also reported increased TA muscle activity related to the decrease in stability, which is caused by wearing HHS during walking (29).

Increasing the muscle activation in patients with FAI during wearing HHS, may be associated with the lateral displacement of the body center of pressure and inversion of the ankle joint before and after heel strike. These changes are considered as important risk factors for ankle instability in patients with FAI (20). So, the increased activation of the TA and PL muscles were expected during unstable positions such as stair descent with HHS. This muscle strategy was also explained by Hopkins et al. (2012) for increasing the TA and PL activity during walking in patients with FAI (20). Accordingly, it has been shown that the limitation in the dorsiflexion in patients with FAI compensated by increasing the TA muscle activation (30). Wearing HHS did not significantly affect the muscle activation in patients with FAI compared with that in healthy subjects with no ready explanation by the authors.

No significant differences were noted between habitual and non-habitual HHS wearers during stair descent with regards to the muscle activation. Foster et al. (2012) and Stefanyshyn et al. (2012) studied the effects of long term use of HHS on the kinematics, kinetics, and muscle activity of the ankle joint. They evaluated only healthy habitual HHS wearers while wearing HHS during walking and found an increase in the PL muscle activity, which was similar to our study. They reported that in the presence of an inversion moment, there might be a greater demand on the ankle evertor muscle activation. Similar to our results, they showed no significant increase in the TA muscle activation during walking, possibly due to the fact that the inversion control was shared by the tibialis posterior muscle $(5,31)$. In contrary to our findings, Cronin et al. (2012) and Alkjear et al. (2012) showed that the activity of the TA and Soleus muscles, increased in healthy habitual HHS wearer during walking. They linked their results to co-activation of the TA and Soleus muscle in habitual HHS wearers $(1,32)$. But in the present study, habitual healthy group don't display a significant increase in TA muscle activation. One possible explanation for this controversy could be the different height of the HHS and the walking task compared to the present research. Similar to our study, Ingrid et al. (2014) attributed the high recruitment of TA muscle in non-habitual HHS wearers to the improvement of instability caused by wearing heels (33).

Considering the points above, findings from this study and those reported previously indicate that some muscle activation changes occur with increases to heel height. This could indicate a greater likelihood of injury or pain in infrequent wearers of HHS, and when wearing HHS for the first time. However, further research is required to confirm this.

\section{CONCLUSIONS}

In conclusion, the findings of the current study identified that activation and onset latencies of the TA, PL, and GM muscles increased with wearing HHS in both healthy and patients with FAI. Therefore, HHS may put people with FAI and without experience of wearing HHS at greater risk for developing ankle sprains.

\section{Limitation}

There are some limitations in this study that can be improved. We did not use electro goniometer or accelerometer to definitely compare the onset times of muscles. So, we had to use the first activated muscle as zero point to estimate muscle latency. Comparing the affected limb with unaffected side in patients with FAI is another limitation in this study. We also did not test shoes with different diameters of the heel to the ground in participants. Future studies are suggested to overcome these limitations.

\section{ACKNOWLEDGMENTS}

The present study was supported by Tabriz University of Medical Sciences.

\section{CONFLICT OF INTERESTS}

The authors declare that they have no conflict of interests. 


\section{REFERENCES}

1. Alkjær T, Raffalt P, Petersen NC, Simonsen EB. Movement Behavior of High-Heeled Walking: How Does the Nervous System Control the Ankle Joint during an Unstable Walking Condition? PLoS ONE 2012;7(5):1-8.

2. Gefen A, Megido-Ravid M, Itzchak Y, Arcan M. Analysis of muscular fatigue and foot stability during high-heeled gait. Gait Posture 2002;15:56-63.

3. Gerber SB, Costa RV, Grecco LA, Pasini H, Marconi NF, Oliveira CS. Interference of high-heeled shoes in static balance among young women. Hum Mov Sci 2012;31:1247-52.

4. Zhang B, Li S, Zhang Y. Evaluation of Dynamic Posture Control when Wearing High-Heeled Shoes Using Star Excursion Balance Test. J Phys Act Health 2017;1(1):1-7.

5. Stefanyshyn D, Nigg B, Fisher V, O’Flynn B, Liu W. The Influence of High Heeled Shoes on Kinematics, Kinetics, and Muscle EMG of Normal Female Gait. J Appl Biomech 2000;16:309-19.

6. Esenyel M, Walsh K, Walden JG, Gitter A. Kinetics of highheeled gait. J Am Podiatr Med Assoc 2003;93(1):27-32.

7. Simonsen EB, Svendsen MB, Nørreslet A, et al. Walking on High Heels Changes Muscle Activity and the Dynamics of Human Walking Significantly. J Appl Biomech 2012;28:20-8.

8. Csapo R, Maganaris CN, Seynnes OR, Narici MV. On muscle, tendon and high heels. Exp Biol 2010;213:2582-8.

9. Benedetti M, Agostini V, Knaflitz M, Bonato P. Muscle Activation Patterns During Level Walking and Stair Ambulation. Applications of EMG in Clinical and Sports Medicine 2012:117-30.

10. Shang J, Chen L, Zhang S, et al. Influence of high-heeled shoe parameters on biomechanical performance of young female adults during stair ascent motion. Gait Posture 2020;81:159-65.

11. Demura T, Demura S, Shin S. Comparison of gait properties during level walking and stair ascent and descent with varying loads. Health 2010;2:1372-6.

12. Hsue B, Sue F. Kinematics and kinetics of the lower extremities of young and elder women during stairs ascent while wearing low and high-heeled shoes. J Electromyogr Kinesiol 2009;19:1071-8.

13. Kim N-H, Choi B-r. The lower-extremity muscle co-activation of flat-footed subjects wearing high-heels while descending stairs. Journal of the Korea Convergence Society 2018;9(11):385-91.

14. Mazaheri M, Salavati M, Negahban H. Reliability and validity of the Persian version of Foot and Ankle Ability Measure (FAAM) to measure functional limitations in patients with foot and ankle disorders. Osteoarthritis Cartilage 2010;18:755-9.

15. Faul F, Erdfelder E, Lang A-G, Buchner A. G* Power 3: A flexible statistical power analysis program for the social, behavioral, and biomedical sciences. Behav Res Methods 2007;39(2):175-91.

16. Padulo J, Oliva F, Frizziero A, Maffulli N. Muscles Ligaments Tnedons Joirnal - Basic principles and recommendations in clinical and field science research: 2018 update. Muscle Ligaments Tendons J 2018;8(3):305-7.

17. Perotto AO. Anatomical guide for the electromyographer: the limbs and trunk (4th ed.). Springfield, Illinois: Charles C Thomas; 2009.
18. Sinclair J, Brooks D, Butters B. Effects of different heel heights on lower extremity joint loading in experienced and in-experienced users: a musculoskeletal simulation analysis. Sport Sci Health 2019;15(1):237-48.

19. Wikstrom EA, Hass CJ. Gait termination strategies differ between those with and without ankle instability. Clin Biomech 2012;27:619-24.

20. Hopkins JT, Coglianese M, Glasgow P, Reese S, Seeley MK. Alterations in evertor/invertor muscle activation and center of pressure trajectory in participants with functional ankle instability. J Electromyogr Kinesiol 2012;22:280-5.

21. Herzog W, Clark A, Wu J. Resultant and local loading in models of joint disease. Arthritis Care Res 2003;49(2):239-47.

22. Zachazewski JE, Riley PO, Krebs DE. Biomechanical analysis of body mass transfer during stair ascent and descent of healthy subjects. J Rehabil Res Dev 1993;30:412-22.

23. Hodges PW, Richardson CA. Inefficient muscular stabilisation of the lumbar spine associated with low back pain: a motor control evaluation of transversus abdominis. Spine 1996;21(22):2640-50.

24. Hodges PW, Richardson CA. Delayed postural contraction of transverse abdominus in low back pain associated with movement of the lower limb. J Spinal Disorders 1998;11(1):46-56.

25. Barton CJ, Coyle JA, Tinley P. The effect of heel lifts on trunk muscle activation during gait: A study of young healthy females. J Electromyogr Kinesiol 2009;19:598-606.

26. Árnadóttir A KI, Magnúsdóttir SK. Electromyographic measurements of walking in high-heeled shoes compared to walking in trainers. University of Iceland 2011.

27. Kermani M, Ghasemi M, Rahimi A, Khademi-Kalantari K, Akbarzadeh-Bghban A. Electromyographic changes in muscles around the ankle and the knee joints in women accustomed to wearing high-heeled or low-heeled shoes. J Bodyw Mov Ther 2018;22(1):129-33.

28. Park J-W, Jee Y-S, Eun D, et al. The effect of wearing highheeled shoes on the isokinetic strength of ankle muscles. Isokinet Exerc Sci 2017;25(3):171-8.

29. Su X, Gu Y. EMG in People with Different Heel Height Condition. In: Steele DC (eds). Applications of EMG in Clinical and Sports Medicine. Voume 2. Croatia: InTech; 2012: pp. 109-16.

30. Drewes LK, McKeon PO, Kerrigan DC, Hertel J. Dorsiflexion deficit during jogging with chronic ankle instability. J Sci Med Sport 2009;12:685-7.

31. Foster A, Blanchette MG, Chou YC, Powers CM. The Influence of Heel Height on Frontal Plane Ankle Biomechanics: Implications for Lateral Ankle Sprains. Foot Ankle Int 2012;33:63-9.

32. Cronin NJ, Barrett RS, Carty CP. Long-term use of high-heeled shoes alters the neuromechanics of human walking. J Appl Physiol 2012;112(6):1054-8.

33. do Nascimento NIC, Sepêda Saraiva T, da Cruz Jr ATV, da Silva Souza, Callegari B. Barefoot and High-Heeled Gait: Changes in Muscles Activation Patterns. Health 2014;6:2190-6. 